\title{
REINGENIERÍA DEL RPAS PISKO V1 DE LA FAP CON TECNOLOGÍA DE PUNTA.
}

${ }^{1}$ Hernando Jose Silva Velasquez
Melgar Gomez, ${ }^{1}$ Julio Curi Arce

${ }^{1}$ Centro De Investigación Y Desarrollo De Proyectos, Fuerza Aérea Del Perú. Lima, Perú

Recibido: 05/09/2021 Revisado: 03/10/2021 Aceptado: 16/11/2021 Publicado: 30/01/2022

\section{RESUMEN}

Los Remote Pilot Aircraft System (RPAS) son una tecnología muy usada actualmente por las Fuerzas Armadas en todo el mundo. En nuestro país, La Fuerza Aérea del Perú (FAP) tiene como unidad tecnológica al Centro de Investigación y Desarrollo de Proyectos (CIDEP) desde el año 2008, que es pionera en el Perú en el desarrollo de proyectos como el RPAS "PEGASO" con bastante éxito en su diseño y operación. Actualmente, seguimos avanzando en el desarrollo de nuevos sistemas, lo que nos ha permitido ser referente dentro de las Fuerzas Armadas de la República del Perú demostrando mejoras continuas en nuestros procesos. Siendo, el objetivo general del proyecto innovar en la reingeniería del RPAS PISKO V1 desarrollando a la versión PISKO V2 cuyas ventajas generan disminución de costos, confiabilidad en la operación y reducción de peso/volumen del equipo en tierra mediante el uso de nuevas tecnologías que cumplen con la seguridad y sostenibilidad haciéndolo más versátil. Los nuevos desafíos operativos de los RPAS en la FAP requieren que estos cuenten con una mejor tecnología para realizar tareas de vigilancia y reconocimiento en tiempo real dentro del territorio Peruano. Por lo tanto, El objetivo principal de este artículo es explicar cómo las nuevas tecnologías permiten realizar mejoras continuas que integren en un solo equipo todos los medios de comunicación (radio control, telemetría y video) para cumplir con los requisitos técnicos y operativos que los RPAS de la FAP necesitan.

Palabras Claves: Reingeniería, RPAS, CIDEP, FAP, vigilancia, reconocimiento, 


\section{ABSTRACT}

The Remote Pilot Aircraft System (RPAS) is a technology widely used today by the Armed Forces around the world. In our country, the Peruvian Air Force (FAP) has as a technological unit the Project Research and Development Center (CIDEP) since 2008, which is a pioneer in Peru in the development of projects such as the RPAS "PEGASO" quite successfully in its design and operation. Currently, we continue to advance in the development of new systems, which has allowed us to be a benchmark within the Armed Forces of the Republic of Peru, demonstrating continuous improvements in our processes. Being, the general objective of the project to innovate in the reengineering of the RPAS PISKO V1 developing the PISKO V2 version whose advantages generate cost reduction, reliability in the operation and weight/volume reduction of the ground equipment through the use of new technologies that meet with safety and sustainability making it more versatile. The new operational challenges of the RPAS in the FAP require that they have better technology to perform surveillance and reconnaissance tasks in real time within the Peruvian territory. Therefore, the main objective of this article is to explain how new technologies allow continuous improvements that integrate all means of communication (radio control, telemetry and video) in a single team to meet the technical and operational requirements that RPAS of the FAP need.

Keywords: Reengineering, RPAS, CIDEP, FAP, surveillance, reconnaissance,

\section{INTRODUCCIÓN}

Nuestras actividades se encuentran bajo la línea de Investigación, Desarrollo e Innovación Tecnológica (I+D+i) que son reguladas con las Normas Técnicas Peruanas (NTP) series 732 y están encargadas de estandarizar la gestión en el campo de la ciencia y tecnología aeroespacial permitiéndonos participar en el Ejercicio de Entrenamiento de Capacidades Operacionales Disimilares ECODEX VII-2018 y en apoyo al Instituto Nacional de Defensa Civil (INDECI) en el simulacro del 2019 transmitiendo en tiempo real en ambos casos. 
Además, contamos con la certificación en la disciplina de investigación de Ingeniería de Sistemas y Comunicaciones con RESOLUCION SUB-DIRECTORIAL N²131-2021CONCYTEC-SDCCTT.

La reingeniería del RPAS PISKO V1 a la versión PISKO V2 permite: i) mejorar la tecnología actual para que pueda operar en condiciones adversas, ii) reducir la cantidad de equipos actuales para mejorar su operatividad mediante el empleo de un sistema integrado; y iii) mejorar la toma de decisiones con la capacidad de información en tiempo real, ya que, estos cuentan con una mejor tecnología para realizar tareas de vigilancia y reconocimiento dentro del territorio Peruano.

Finalmente, este artículo académico detallará el proceso de elección de la tecnología y reingeniería aplicada al RPAS PISKO V1 para cumplir con el objetivo de la versión V2 para vigilancia y reconocimiento.

\section{ELECCION DE LA TECNOLOGIA DE PUNTA}

Como mencionamos al inicio de este artículo, el CIDEP es pionera en el desarrollo de RPAS en el país y siempre anda buscando mejoras tecnológicas para sus nuevos sistemas. Aplicaciones de vigilancia y reconocimiento en tiempo real es de las más requeridas para la seguridad de bases aéreas, lucha contra el narcotráfico, minería ilegal, deforestación, contrabando y seguridad ciudadana.

En el mercado existen muchos drones comerciales para vigilancia y reconocimiento, estos tienen un rango de alcance, autonomía, sensores y peso muy parecidos o mejores al RPAS PISKO V1 y además cuentan con equipos más compactos que lo hacen más prácticos a la hora de operar. El precio de estos RPAS pueden variar pero uno de los más populares es el Phantom 4 Pro V2 de la empresa DJI a un precio en el mercado local de aprox. S/. 7,900.00

DJI Phantom 4 Pro

Peso $1,388 \mathrm{gr}$ 


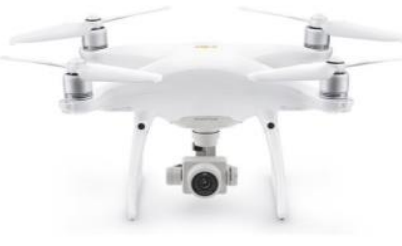

\begin{tabular}{|l|l|}
\hline Max. Altitud & $6,000 \mathrm{~m}$ \\
\hline Autonomía & $30 \mathrm{~min}$ \\
\hline Cámara & $20 \mathrm{M}$ \\
\hline Gimbal & 3 ejes \\
\hline Max. Transmisión & $5 \mathrm{Km}$ \\
\hline
\end{tabular}

Por otra parte, el RPAS PISKO V1 desarrollado por el CIDEP el año 2019 contaba con características muy parecidas.

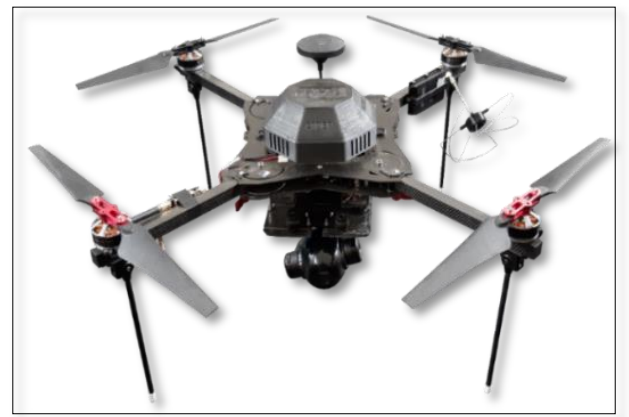

\begin{tabular}{|l|l|}
\hline \multicolumn{2}{|l|}{ RPAS PISKO V1 } \\
\hline Peso & $2,800 \mathrm{gr}$ \\
\hline Max. Altitud & $3,500 \mathrm{~m}$ \\
\hline Autonomía & $20 \mathrm{~min}$ \\
\hline Cámara & $\mathrm{HD}$, Zoom 10X, \\
\hline Gimbal & 3 ejes \\
\hline Max. Transmisión & $5 \mathrm{Km}$ \\
\hline
\end{tabular}

Pero su Estación de Control no era muy practica para las operaciones al tener los enlaces de comunicación separado para radiocontrol, telemetría y video, como se muestra en la Figura X. 


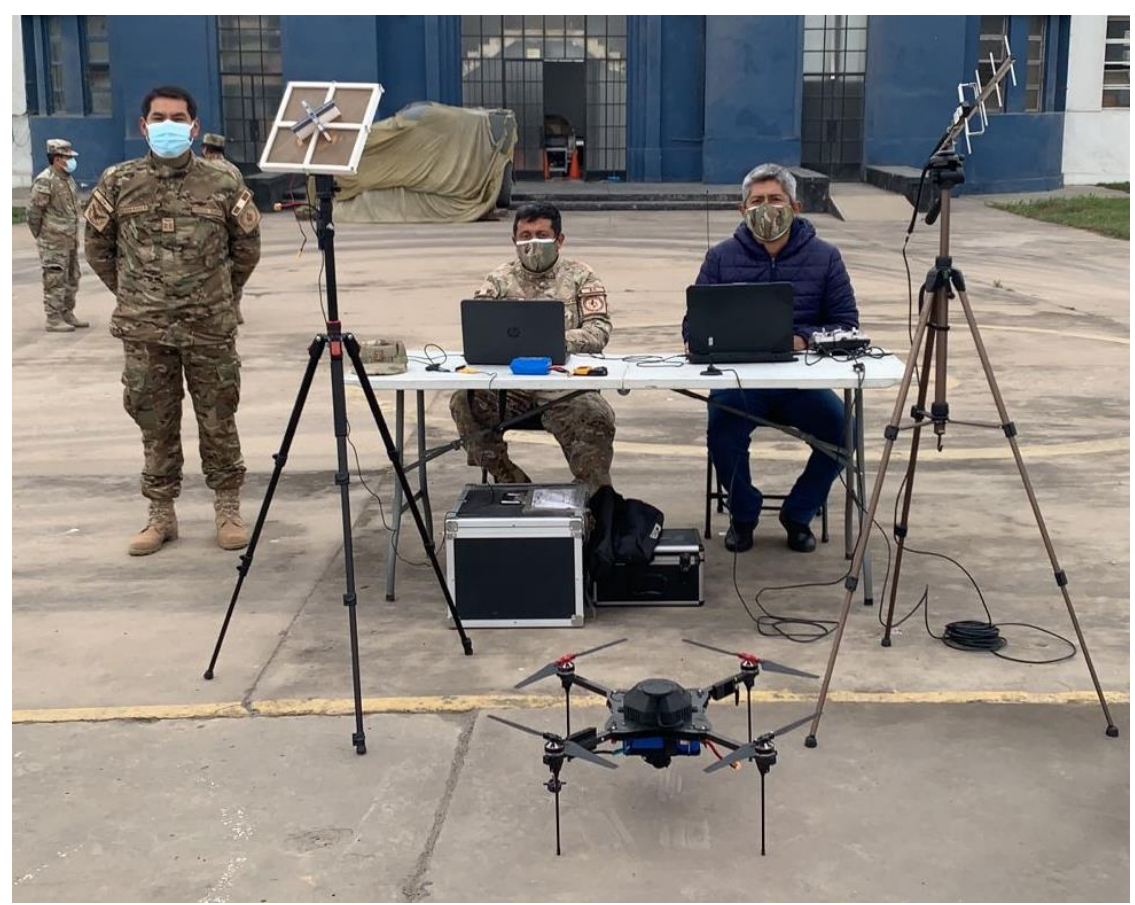

En el año 2020, el CIDEP propone hacer una reingeniería al RPAS PISKO V1, considerando mejoras en su autonomía, facilidad de operación e integración de todos los enlaces de comunicación.

\begin{tabular}{|l|l|}
\hline HERELINK & \\
\hline Rango de Transmisión & $\begin{array}{l}\text { Default 12Km y Max 20 } \\
\text { Km }\end{array}$ \\
\hline Telemetría & RC, Data y Video \\
\hline Frecuencia & 2.4GHz \\
\hline Protocolo datos & UART/Mavlink \\
\hline Video & HDMI x 2 \\
\hline
\end{tabular}
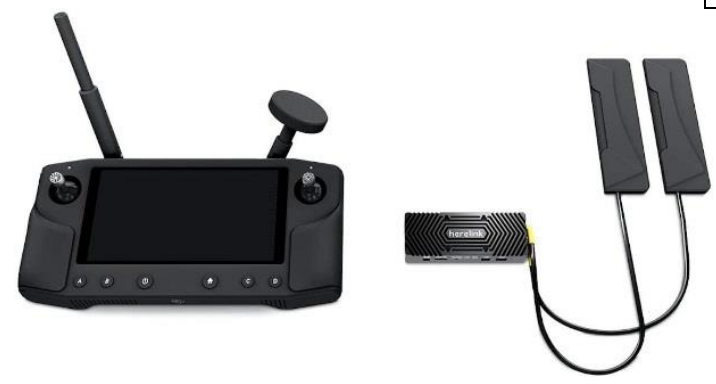

BIOTECH \& ENGINEERING Untels. Ene -Jun.2(1), 2022; ISSN:2788 -4295; 345-351 
Con esta nueva tecnología, se descarta el uso de las antenas de datos, video y el antiguo radio control Taranis. Se cambian las antenas aéreas y terrestres, dando lugar a un solo equipo compacto que integra todo.

Se puede ver en la imagen como solo es necesario el operador y su mecánico para la operación, en comparación a como se operaba con la versión anterior. 


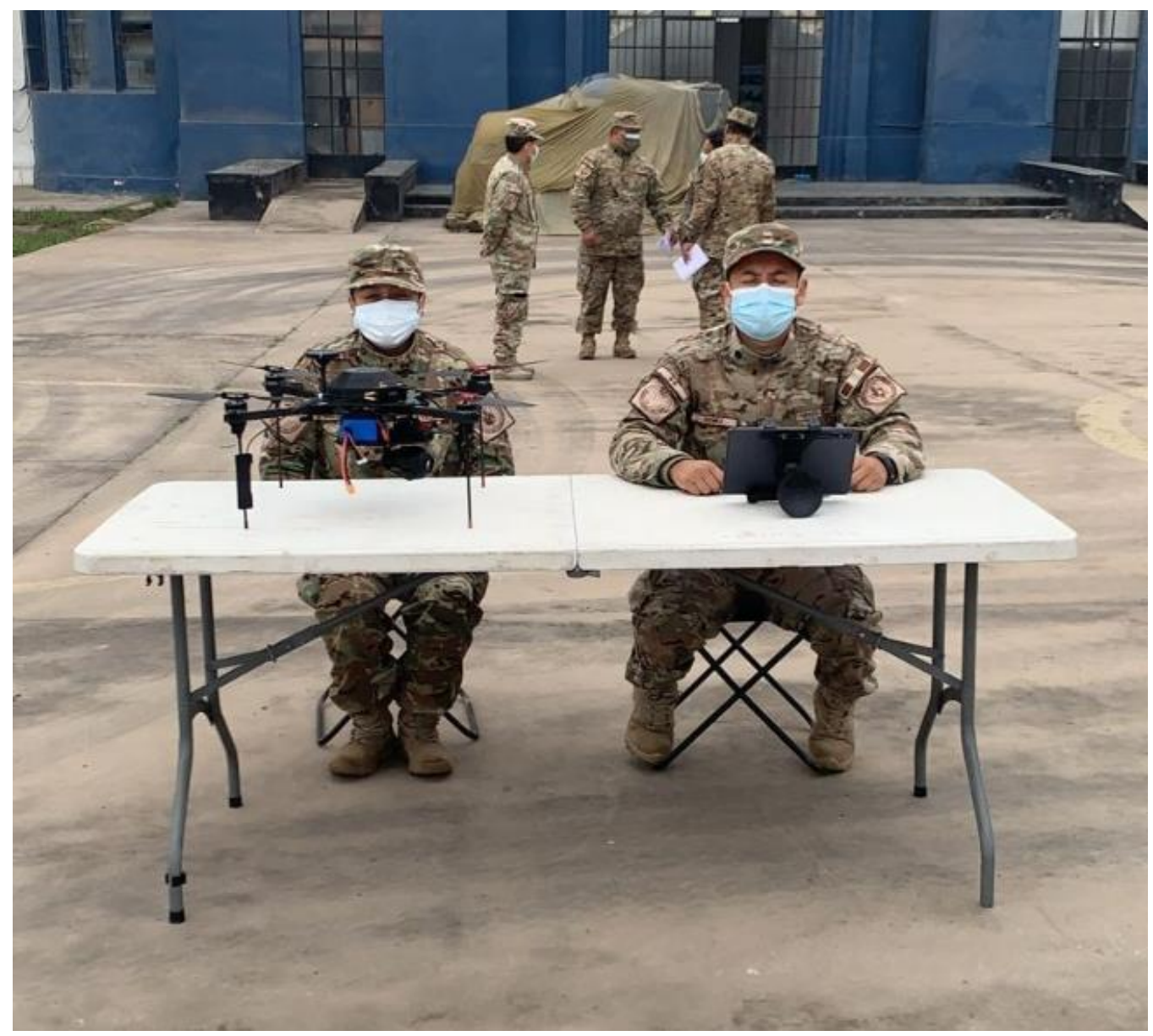

\section{REFERENCIA}

[1] URL: https://www.defensa.com/peru/ecodex-viii-fuerza-aerea-peru-pone-pruebacapacidades-galeria 